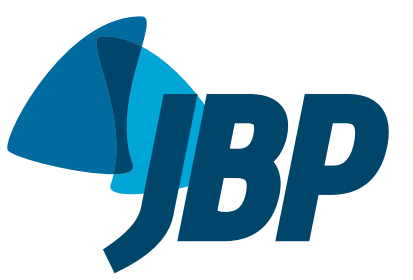

A 41-year-old male presented with complaints of anorexia and weight loss followed by cough with hemoptysis. The final diagnosis was aspergilloma in previous tuberculous lesions.

The finding of a nodule in a lung cavity has important diagnostic and therapeutic implications. Although aspergilloma is the most common cause of intracavitary nodules, a number of other conditions should be included in the differential diagnosis. These conditions primarily include neoplasms (particularly bronchial carcinoma), angioinvasive aspergillosis during the recovery phase, Rasmussen aneurysm, and clots. Other, rarer, causes include foreign bodies, thick pus, dehydrated caseous material, teratoma, and hydatidosis. The air crescent sign is commonly seen in patients with intracavitary nodules, regardless of the etiology. It corresponds to a collection

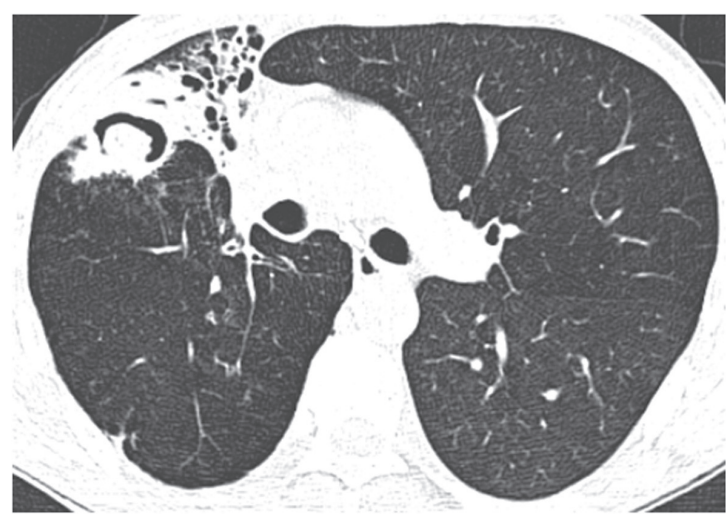

Figure 1. CT scan of the chest with lung window settings at the level of the main pulmonary artery showing volume loss in the right upper lobe, as well as bronchiectasis and a cavitary lesion containing a nodular density. Note air interposed between the nodule and the cavity wall (the air crescent sign) of air in the form of a crescent or half moon, located in the periphery of a nodule or mass of soft tissue density, separating the nodule from the cavity wall.

A useful imaging criterion for the differential diagnosis is a change in the position of the nodule in the cavity when patient position is changed, especially during examination in the supine and prone positions. It is extremely important to determine whether the central mass is free or attached to the cavity wall because, unlike a fungus ball or a clot, cavitary lung cancer and Rasmussen aneurysm present as masses that are fixed to the cavity wall; that is, they do not move when patient position is changed. Contrast enhancement of the nodule on CT scans can aid in differentiating between aspergilloma and Rasmussen aneurysm. In cases of Rasmussen aneurysm, which is a pulmonary artery pseudoaneurysm secondary to pulmonary tuberculosis, hemoptysis is a common initial manifestation and can be fatal when it is massive. However, hemoptysis is also a common finding in patients with aspergilloma.

A fungus ball or aspergilloma is the most common cause of intracavitary nodules, generally resulting from fungal colonization of pre-existing lung cavities. Although such cavities are most commonly due to tuberculosis, fungus balls can develop in cysts, bullae, and bronchiectasis. Colonization with Aspergillus spp. occurs in most cases, which is why the term "aspergilloma" is commonly used. However, the air crescent sign has been reported in association with other fungal infections and bacterial infections, including coccidioidomycosis, actinomycosis, nocardiosis, and candidiasis. In conclusion, although aspergilloma is the most common cause of intracavitary nodules, other conditions should be considered in the differential diagnosis, including intracavitary tumors and Rasmussen aneurysm.

\title{
RECOMMENDED READING
}

1. Fraser RS, Muller NL, Colman NC, Pare PD, editors. Fraser and Pare's Diagnosis of Diseases of the Chest. 4th ed. Philadelphia: Saunders; 1999. 